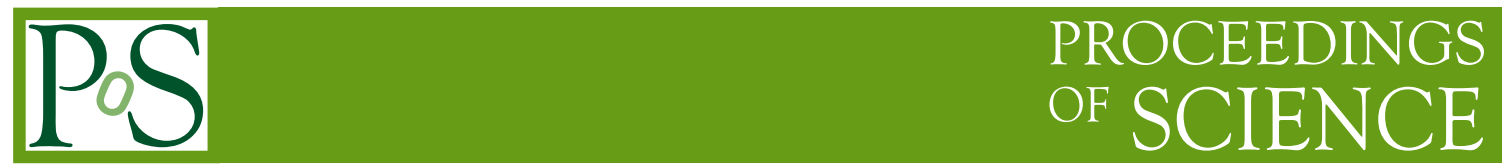

\title{
The status of INO
}

\author{
Amitava Raychaudhuri*† \\ Harish-Chandra Research Institute \\ Chhatnag Road, Jhunsi, Allahabad 211 019, India. \\ E-mail: raychaud@hri.res.in
}

A brief status report of the India-based Neutrino Observatory is presented. Its physics goals are surveyed.

10th International Workshop on Neutrino Factories, Super beams and Beta beams

June 30 - July 52008

Valencia, Spain

* Speaker.

${ }^{\dagger}$ (on behalf of the INO collaboration) 


\section{Introduction}

The India-based Neutrino Observatory (INO) is a proposal, initiated in 2002, to set up a lowbackground facility with emphasis on neutrino physics. The first experiment being planned at INO is with a magnetized iron detector - an iron calorimeter (ICAL). The laboratory may also house experiments in other disciplines that can profit from its special environment and infrastructure.

India has a good track record in non-accelerator particle physics; the signature of the $v_{\mu}$ in cosmic-rays was first noted by an experiment based in India and the world's first dedicated proton decay experiment was located at the Kolar Gold Fields [1].

In this abridged version of the Workshop presentation, focussed on ICAL, we will sample some possible physics prospects and then turn to a status report on the detector and simulation [2].

\section{Physics prospects}

ICAL will start as a 50kTon magnetized iron calorimeter with Resistive Plate Chambers (RPCs) as the active detector elements. Atmospheric muon neutrinos/antineutrinos above a threshold of $1 \mathrm{GeV}$ will be detected via the muon tracks in the detector. It may also be used to detect ultra-high energy muons through the $e^{+} e^{-}$pair counting technique.

The immediate goal of ICAL will be a confirmation of the oscillation in the number of events as a function of the zenith angle (or $L / E$ ) and to extract the mixing parameters. The variation of the ratio of the number of upgoing neutrinos to the number of downgoing neutrinos $(U / D)$ captures the oscillatory behaviour, reducing the systematic uncertainties. Simulations indicate that this method will allow this initial goal to be achieved well within the first 5-year run.
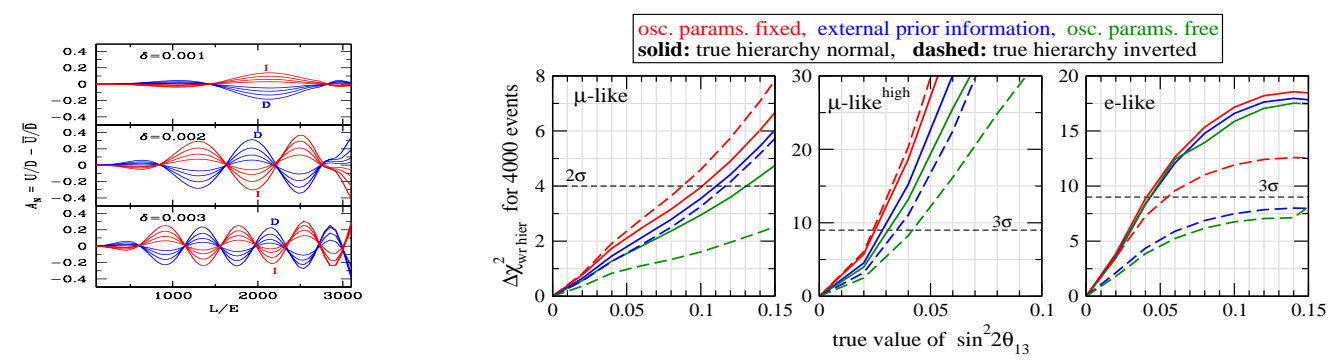

Figure 1: Determining the neutrino mass ordering from atmospheric neutrinos at ICAL (see text). See $[2,4]$.

Since matter interactions affect neutrinos and antineutrinos differently, their $U / D$ ratio will not be the same and, in particular, the asymmetry $A_{N}=(U / D)-(\bar{U} / \bar{D})$ will signal the ordering of neutrino masses. This is exhibited in Fig. 1 (left) for three different choices of $\left|\Delta_{31}\right| \equiv \delta$ (in $\mathrm{eV}^{2}$ ). The red (blue) curves correspond to normal (inverted) hierarchy and the innermost (outermost) curves correspond to $\theta_{13}=5^{\circ}\left(11^{\circ}\right)$ (see also [3]).

A detailed analysis for the determination of the neutrino mass ordering through atmospheric neutrinos in an ICAL-type magnetized calorimeter can be found in [4]. Some of their findings are shown in Fig. 1 (right) assuming a data sample of 4000 events. Here the impact of energy and angular resolutions of the detector (5\% in the middle panel and $15 \%$ in the others) and other uncertainties have been carefully included. 
It is seen from Fig. 1 (right) that the sensitivity to the neutrino mass ordering falls sharply with $\theta_{13}$. A recent noteworthy study indicates that at very small (or even vanishing) values of $\theta_{13}$, focussing on $v, \bar{v}$ with $E \sim 1 \mathrm{GeV}$ and $L \sim 10^{4} \mathrm{kms}$ it might be possible to find the sign of $\Delta_{31}$ at the $3 \sigma$ level with 1MTon-year data if $\left|\Delta_{31}\right|$ itself is determined to a high precision [5].

The current best-fits of $\theta_{23}$ are centred around $\pi / 4$, which corresponds to maximal mixing. Many models of neutrino masses lead to maximal mixing in this sector. It is of interest to experimentally establish whether there is any deviation of $\sin 2 \theta_{23}$ from maximality. With 1MTonyear/3.37MTon-year data, an ICAL-based study of atmospheric neutrinos could indeed tell whether $\sin 2 \theta_{23}$ is truly maximal [6]. The results for $\sin ^{2} \theta_{13}=0.0,0.02$, and 0.04 are shown in Fig. 2 (left). The different shadings correspond to $1 \sigma, 2 \sigma$, and $3 \sigma$ excluded regions.
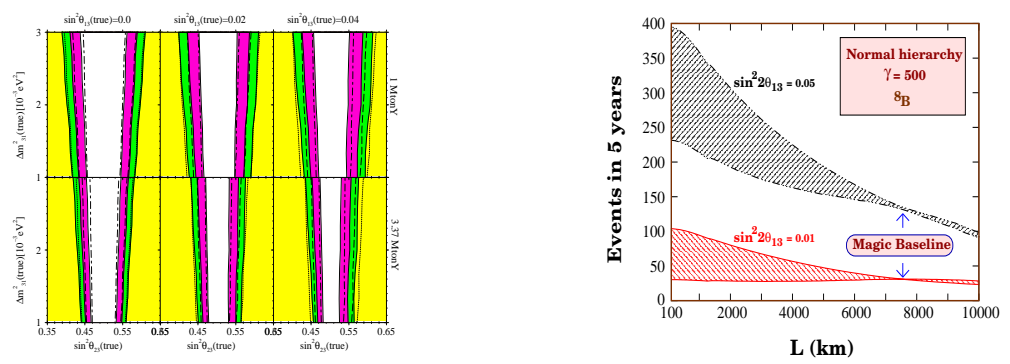

Figure 2: Addressing the octant amibuguity (left) and Magic baseline behaviour (right). See [6, 7].

In a second phase ICAL may be used as the end-detector for a long-baseline experiment with a beta-beam/neutrino factory source in Europe. The distance is close to the "magic" baseline where the dependence on the unknown leptonic CP-phase, $\delta$, is small. This property of the baseline is illustrated in Fig. 2 (right) where the number of events in five years from a beta beam source is plotted as a function of the baseline. The width of the band for any $L$ is an effect of the unknown $\delta$. The $\delta$-independence permits an unprecedented precision in the determination of the neutrino mass ordering and $\theta_{13}$ for a magic baseline experiment [7].

\section{INO: status today}

INO will be built in a cavern $-26 \mathrm{~m}(\mathrm{w}) \times 25 \mathrm{~m}(\mathrm{~h}) \times 132 \mathrm{~m}(\mathrm{l})-$ inside a mountain with good access by road tunnels. A suitable location providing $1 \mathrm{~km}$ rock coverage (2.8km water equivalent) from all directions has been identified (PUSHEP, Latitude: $\mathrm{N} 11.5^{\circ}$, Longitude: E76.6 ${ }^{\circ}$ ) in the Nilgiris, about 250km from Bangalore - see Fig. 3 (left). Geological and environmental studies have progressed satisfactorily. An INO Centre located in Mysore, about $90 \mathrm{~km}$ from the site, will be the base for many of the INO scientists, will house detector and electronics laboratories, and will be in charge of training and outreach programmes.

The experiment is planned to start in about five years. An INO project report submitted to the Government of India [2] was internationally reviewed and recommended. Funding for INO has been approved 'in principle' and release of funds is awaiting further clearances at different levels of government.

The proposed detector, see Fig. 3 (centre), will have three modules of lateral sizes $16 \mathrm{~m} \times$ $16 \mathrm{~m}$ each and a height of about $12 \mathrm{~m}$. This is composed of 140 layers of iron plates of thickness 

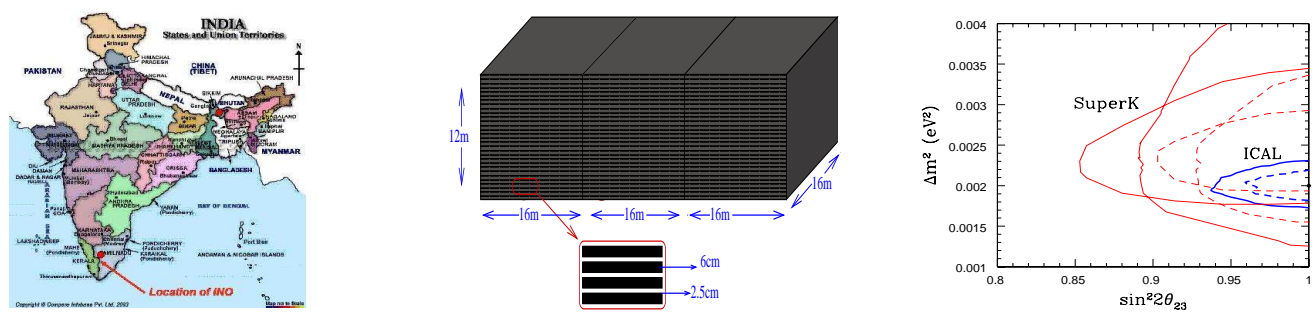

Figure 3: The location of INO (left), a sketch of the ICAL detector (center), and simulated results for oscillation parameters from ICAL (right).

$6 \mathrm{~cm}$ each, inter-leaved with active detector elements - an RPC placed in a gap of $2.5 \mathrm{~cm}$ between the plates. The iron plates can be magnetized up to 1-1.3Tesla. The total mass of such a detector including the support structure etc, will be approximately 50kTon.

Prototype $1 m \times 1 m$ glass RPCs with graphite coating have been fabricated at the Tata Institute of Fundamental Research (TIFR), Mumbai and are being tested for long-term stability in the avalanche mode. Bakelite RPCs with silicone coating running in the streamer mode are being examined at the Saha Institute of Nuclear Physics, Kolkata. A major milestone has been crossed with the efficiency of the RPCs at TIFR surpassing $90 \%$ above $8.6 \mathrm{kV}$. The timing for the prototype RPCs is found to be better or as good as in the case of a scintillator detector. The design for the ICAL magnetic field is nearing completion.

Work on the ICAL detector simulation is in active progress. The first goal of the experiment will be to extract precise information about neutrino masses and mixings using atmospheric neutrino events. A GEANT3-based ICAL simulation program incorporating the magnetic field has been used to explore the energy and angular resolutions, charge identification efficiency, etc. and to identify appropriate cuts for event reconstruction. For this simulation atmospheric neutrino events with the Honda flux are generated using the NUANCE package. With a threshold of $1 \mathrm{GeV}$, the detection efficiency is found to be $87 \%$ and charge identification efficiency is $94 \%$ for fullycontained (FC) events. Sample results for 300kTon-years FC events at ICAL are compared with SuperK zenith angle and $L / E$ findings in Fig. 3 (right) where the solid (broken) curves are for 99\% $(90 \%)$ CL. Currently, work is in progress on a GEANT4 version of ICAL simulation.

To summarise, the main characteristics of the ICAL detector are a clean identification of muons with good energy, position, and time resolution with charge identification capability.

A 40Ton prototype of ICAL consisting of 13 layers of $5 \mathrm{~cm}$ thick magnetized iron plates is being set-up at the Variable Energy Cyclotron Centre, Kolkata. The iron for this purpose and the magnet coils (field $\sim 1$.5Tesla) have already been delivered and installed in the test-area. Fabrication of glass and bakelite RPCs for this detector are underway. The prototype will be tested first with atmospheric neutrinos and later possibly at a test beam facility.

\section{Outlook}

The INO project and the 50kTon magnetized iron ICAL detector, which will probe the neutrino mass spectrum and mixing angles to high precision, are being vigorously pursued by a team of scientists and engineers. The project has been cleared at various levels and is now awaiting the 
release of already earmarked funds by the government. The site for INO has been identified and DPR for the site and infrastructure are in an advanced stage of preparation. The first batch of INO students have been selected and they are undergoing intensive training. Glass and bakelite RPCs have been fabricated and are being tested for long-term stability. An ICAL prototype is almost ready for trial.

In a second phase the detector is planned to be enlarged to 100kTons. The location of INO at a distance close to the "magic" baseline from Europe will make it an attractive far-detector for beta-beam/neutrino factory sources in Europe.

The INO collaboration welcomes participation from all over the world.

\section{ACKNOWLEDGEMENTS:}

This work has been supported by the XIth Plan 'Neutrino' project at HRI.

\section{References}

[1] C.V. Achar, M.G.K. Menon, V.S. Narasimham, et al., Detection of Muons Produced by Cosmic Ray Neutrino Deep Underground, Phys. Lett. 18 (1965) 196; M.R. Krishnaswamy, M.G.K. Menon, N.K. Mondal, et al., Candidate events for nucleon decay in the Kolar Gold Field experiment, Phys. Lett. 106 (1981) 339.

[2] Details of the INO proposal and the substantial research on its physics reach may be found in the INO Project Report INO/2006/01 at http://www.imsc.res.in/ ino.

[3] D. Indumathi and M.V.N. Murthy, A question of hierarchy: Matter effects with atmospheric neutrinos and anti-neutrinos, Phys. Rev. D 71 (2005) 013991 [hep-ph / 0407336$].$

[4] S.T. Petcov and T. Schwetz, Determining the neutrino mass hierarchy with atmospheric neutrinos, Nucl. Phys. B 740 (2006) 1-22 [hep-ph/0511277].

[5] Raj Gandhi, Pomita Ghoshal, Srubabati Goswami, and S. Uma Sankar, Mass hierarchy determination using atmospheric neutrinos for small $\theta_{13}$, [arXiv: 0805.3474].

[6] Sandhya Choubey and Probir Roy, Probing the deviation from maximal mixing of atmospheric neutrinos, Phys. Rev. D 73 (2006) 013006 [hep-ph / 0509197$].$

[7] S.K. Agarwalla, A. Raychaudhuri, and A. Samanta, Exploration prospects of a long baseline beta beam neutrino experiment with an iron calorimeter detector, Phys. Lett. B629 (2005) 33 [hep-ph $10505015] ;$ S.K. Agarwalla, S. Choubey and A. Raychaudhuri, Neutrino mass hierarchy and $\theta_{13}$ with a magic baseline beta-beam experiment, Nucl. Phys. B771 (2007) 1 [hep-ph/ 0610333$]$; Unraveling neutrino parameters with a magical beta-beam experiment at INO, ibid. B798 (2008) 124 [arXiv:0711.1459], and references therein. 